\title{
An unusual case of neuroma of the median nerve
}

\author{
R. KANAGASUNTHERAM AND W. C. WONG \\ From the Anatomy Department, University of Singapore
}

The use of skin biopsy in the investigation of sensory disturbances due to peripheral neuropathies is of recent origin. Dickens, Winkelmann, and Mulder (1963), Dyck, Winkelmann, and Bolton (1966) and more recently Ridley (1968) have made use of such biopsy material to assess the extent of loss of Meissner's corpuscles and have tried to correlate this with the degree of sensory loss in their patients. In none of the cases, however, was there reported the presence of innervated Meissner's corpuscles and nerve endings where there was complete loss of sensation. The only exception was one case of hypertrophic poly-neuropathy, case 51 (Ridley, 1968) where, although there was complete loss of touch sensation, Meissner's corpuscles were still present though very much reduced. Ridley (1968) did not comment further on this case. Besides Ridley's case, such findings have not been reported in the literature as far as the authors are aware, with one proviso, that in leprous skin, nerve endings have been claimed to be present in anaesthetic zones (see Dash, 1968). It was, therefore, thought desirable to report the present unusual findings of a case of neuroma arising after nerve suture in which the skin was totally devoid of touch sensation at the time of biopsy but there were histologically demonstrable innervated Meissner's corpuscles and nerve bundles in the skin.

\section{CASE REPORT}

The patient, W.H., a male Malay labourer aged 21, was admitted to the General Hospital, Singapore, on 3 July 1966 with multiple fractures that included a fracture of the lower end of the left radius for which a manipulative reduction was done. When seen on 14 December 1966 for medico-legal assessment, the patient complained of pain at the wrist and lack of feeling over the left thumb, index and middle fingers and the outer half of the palm. Examination showed a sensory loss over the distribution of the left median nerve and muscle wasting of the left thenar eminence. Radiographs of the wrist showed mal-union of the fracture. The median nerve was explored on 16 December 1966 and found to be fibrotic and caught up in the fracture of the lower end of the left radius. The nerve ends were excised and apposed by silk sutures. The patient resumed light duties on 25 May
1967. When seen again on 17 January 1968 for medicolegal assessment the patient complained of numbness in the left hand and pain at the left wrist. Examination showed an area of anaesthesia on the left palm, the palmar surfaces of thumb, index, middle and half of the ring fingers. The median nerve was re-explored on 23 February 1968 and at the site of the previous nerve suture a neuroma $2 \mathrm{~cm}$ long was found. No muscle movement occurred on electrically stimulating the median nerve. About $3.5 \mathrm{~cm}$ of the median nerve including the neuroma was excised and the nerve ends were then sutured. Two pieces of skin, one from the palmar aspect of the terminal phalanx of the index finger and another from the $N$ thenar eminence, were removed on this occasion fos or study. When seen on 25 September 1968 there was aro $\overrightarrow{0}$ aesthesia over the left thenar eminence with minima sensation present in the first three digits. When the patient was re-examined on 12 December 1968 he show ed remarkable improvement and was able to recogniz $\bigcirc$ fine touch when examined with cotton wool.

NEUROHISTOLOGY A part of the biopsy material was processed for the presence of acetylcholinesterase (AChE using a modified Koelle's technique, while the remaining of portions of the specimens were stained for nerve fibres by the Bielschowsky-Gross method. Some sections of the neuroma were stained by haematoxylin and eosin, while others were processed by the Bodian silver technique for the presence of nerve fibres.

Thenar eminence On examination of the biopsy mat- $\stackrel{\mathbb{D}}{\triangle}$ erial from the thenar eminence occasional nerve bundles $\overrightarrow{\vec{P}}$ consisting of unmyelinated and thinly myelinated fibres were seen in the corium (Fig. 1). A few fibres appeared to be swollen and showed the presence of alternate light and dark bands. Very rarely a few unmyelinated fibres were seen coursing for a short distance parallel to the surface of the epidermis. No nerve fibres were seen terminating in the dermal papillae or among epidermal 0 cells.

Digital skin The innervation here was more abundant than in the thenar eminence. There were normal-looking nerve fibres, both myelinated and unmyelinated, running beneath the epidermis. However, there were also fibres $\frac{}{5}$ which presented a striated, swollen and varicose appear- $\rightarrow$ ance similar to those in the palmar region (Fig. 2). 을. Occasionally nerve fibres were seen in the dermal papilla entering a Meissner's corpuscle (Fig. 3). The nerve $O$ supply to some corpuscles revealed that it was less com- $N$ plex than that which obtains in normal human skin. N Some terminations formed simple loops (Fig. 4), while 


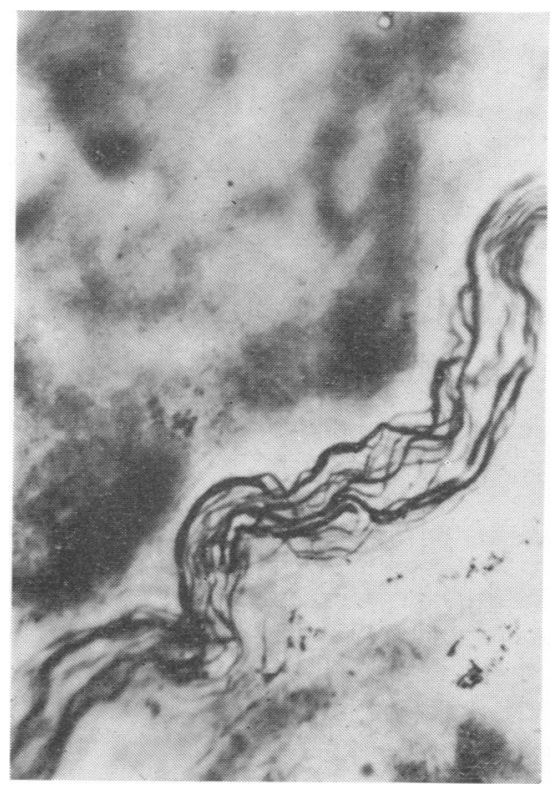

FIG. 1. Human palm showing a bundle of unmyelinated and thinly myelinated nerve fibres in the corium. Bielschowsky-Gross method. $\times 344$.

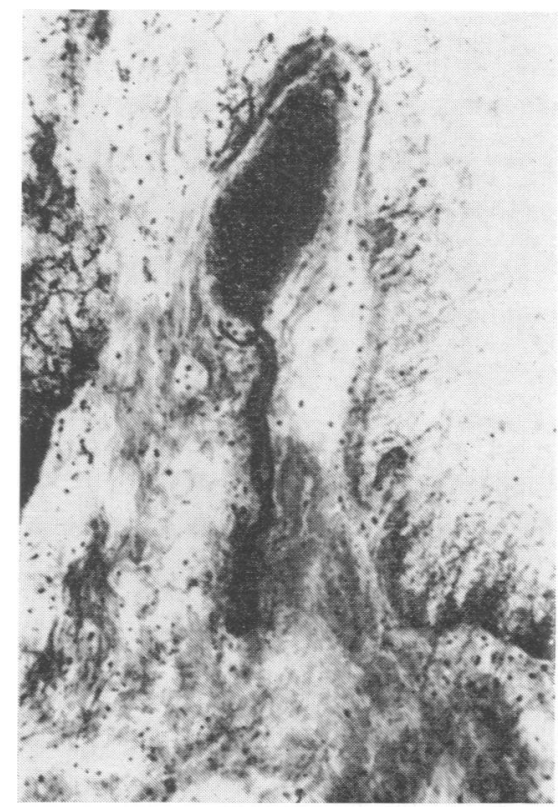

FIG. 3. Human digit showing an innervated Meissner's corpuscle. Bielschowsky-Gross method. $\times 344$.

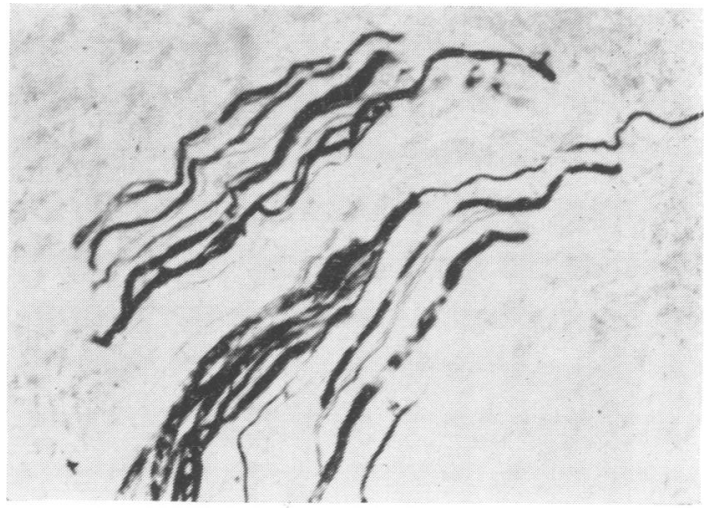

FIG. 2. Human digit showing unmyelinated and thinly myelinated nerve fibres in the corium. Some of the fibres show striations. Bielschowsky-Gross method. $\times 344$.

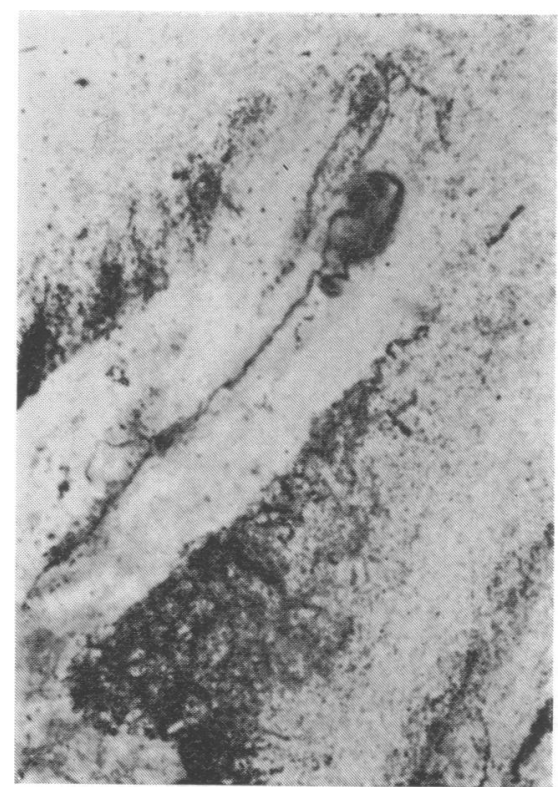

FIG. 4. Human digit showing a nerve fibre in a Meissner's corpuscle forming a simple loop. Bielschowsky-Gross method. $\times 344$. 


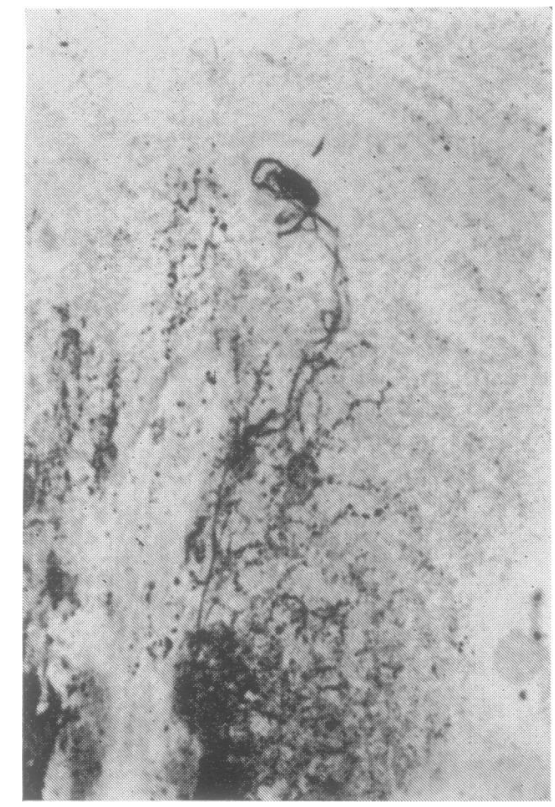

FIG. 5. Human digit showing a nerve fibre in a Meissner's corpuscle breaking up into a neurofibrillar apparatus. Bielschowsky-Gross method. $\times 344$.

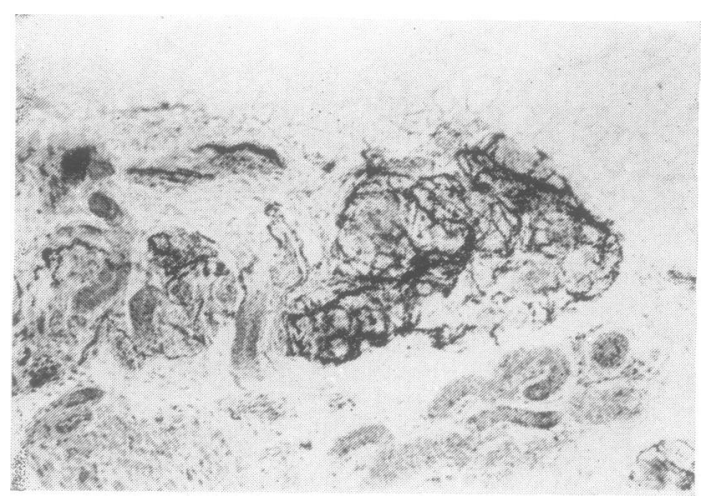

FIG. 6. Sweat gland lobules in human digit showing partial innervation. Koelle's technique for acetylcholinesterase. $\times 86$.

other corpuscles showed breaking up of fibres into a neurofibrillar apparatus (Fig. 5). Still others merely showed dark staining clumps (presumably fibres) in place of the corpuscle. Sometimes both thick and thin fibres were seen passing towards a corpuscle. Some fibres within the corpuscle were heavily stained, while others showed a very poor staining reaction. Moreover, an occasional thin fibre was seen to terminate freely near the apex of a dermal papilla or at the base of an interpapillary ridge.

Histochemistry In the digit a trace of true AChE activity was present in an occasional Meissner's corpuscle, whereas no such activity was observed in the thenar eminence. In the sweat glands there was $\mathrm{AChE}$ activity in some lobules whereas others were devoid of activity (Fig. 6). Unfortunately no pseudo-cholinesterase activity was tested.

Neuroma Examination of the neuroma showed an abundance of nerve fibres and a large amount of fibrous tissue.

\section{DISCUSSION}

The most interesting feature of the present case of neuroma of the median nerve is the presence of nerve terminals including Meissner's corpuscles in the skin biopsy taken over the anaesthetic area. Only Ridley (1968) has reported similar findings in a case of polyneuropathy, but without comment. To our knowledge, in no previous instance was there a skin biopsy taken in a case of neuroma to review the position of nerve terminals. Even a recent publication by Sunderland (1968), while giving a detailed classification of various types of neuroma, has not reported anything similar to the present case.

Although simple nerve terminals as well as Meissner's corpuscles were present in the case undero review these corpuscles with the exception of one $\infty$ or two were not as richly innervated as normal ones? described by Cauna (1956). The nerve terminals $]$ within the corpuscles were rather few and some ofo them formed simple loops similar to those described by Kanagasuntheram and Krishnamurti (1969) ino the foetal skin of some lower primates. Consequently the features of the present case would indicate only a partial reinnervation of the skin after the suture of the nerve and subsequent formation of the neuroma. Thus the picture presented was somewhat similar to that described by Jayaraj and Chaudhury (1960), Dastur (1955) and Dash (1968) in anaesthetic areas of leprous skin where biopsy revealed a few nerve terminations and nerve bundles. Moreover, recent studies by Dash (1968) showed that sensations could be restored over the anaesthetic zones in leprous patients by a combination of antidromic and natural stimuli of the affected nerves. This proves that some unknown nerve factor concerned with the conduction of impulses was probably prevented from passing distally in leprosy. A similar phenomenon rather than mere mechanical pressure due to excess fibrous tissue might be the explanation for the anaesthetic condition obtained in our present case. Another explanation that is possible is that the disorderly growth of fibres in a neuroma might result in inappropriate connections between the severed ends of the nerve fibres. Consequently, the number of remaining normal nerve fibres is insufficient to give rise to a normal pattern of impulses which would elicit sensation. Such an explanation is in 
accord with the views of Weddell, Palmer, and Pallie (1955).

The second point of interest in the present case is the partial retention of cholinergic fibres to some of the sweat glands of the digital skin supplied by the affected median nerve. It is generally accepted that the cholinergic fibres to the sweat glands pass along the digital branches and separate off from these nerves to innervate the glands (Cauna and Mannan, 1961). If this pattern of innervation is accepted, the presence of cholinergic fibres within the territory of the median nerve becomes explicable if it is assumed that only some fibres were affected by the neuroma while others had remained normal. However, it is also possible that even if all the fibres were affected by the neuroma the sweat glands which had retained their cholinergic supply could have received their innervation through another source-namely, from nerves accompanying the blood vessels. That such a distinct possibility exists is shown by our recent experimental studies on the median nerve of monkeys (Kanagasuntheram and Wong, 1969).

Lastly, it is noteworthy that the median nerve remained severed between 3 July 1966 and 15 December 1966. Moreover, the nerve had not been functioning normally between 16 December 1966 and 23 February 1968 when the neuroma had formed after the suture. Thus, in view of the fact that the nerve had remained non-functional for a period of over one and a half years, the recovery of touch sensibility by December 1968 after the re-suture of the nerve in February 1968 is indeed remarkable.
We thank Professor V. K. Pillay (Department of Orthopaedics, University of Singapore) for access to the case and the Director of Medical Services, Singapore, for permission to publish the case records. Our thanks are also due to Mr. Lee Chuan Tee and Mr. Chua Beng Cheng for technical help.

\section{REFERENCES}

Cauna, N. (1956). Nerve supply and nerve endings in Meissner's corpuscles. Amer. J. Anat., 99, 315-350.

the preterminal nerve pattern in the palmar digital tissues of man. J. comp. Neurol., 117, 309-327.

Dash, M. S. (1968). A study of the mechanism of cutaneous sensory loss in leprosy. Brain, 91, 379-392.

Dastur, D. K. (1955). Cutaneous nerves in leprosy; relationship between histopathology and cutaneous sensibility. Ibid., 78, 615-633.

Dickens, W. N., Winkelmann, R. K., and Mulder, D. W. (1963). Cholinesterase demonstration of dermal nerve endings in patients with impaired sensation. Neurology (Minneap), 13, 91-100.

Dyck, P. J., Winkelmann, R. K., and Bolton, C. F. (1966). Quantitation of Meissner's corpuscles in hereditary neurologic disorders. Charcot-MarieTooth disease, Roussy-Levy syndrome, Dejerine-Sottas disease, hereditary sensory neuropathy, spinocerebellar degenerations and hereditary spastic paraplegia. Ibid., 16, 10-17.

Jayaraj, A. P., and Chaudhury, D. S. (1960). Epithelial and subepithelial innervation in lepromatous leprosy. Leprosy in India, 32, 167-169.

Kanagasuntheram, R., and Krishnamurti, A. (1969). Nerve endings in the skin of the foetal hand of slow loris. Folia primatol., 10, 263-275.

nervation of the digital tissues in the hand of Macaca fascicularis. In preparation.

Ridley, A. (1968). Silver staining of the innervation of Meissner corpuscles in peripheral neuropathy. Brain, 91, 539-552.

Sunderland, S. (1968). Nerves and Nerve Injuries. Pp. 180-194. Livingstone: Edinburgh and London.

Weddell, G., Palmer, E., and Pallie, W. (1955). Nerve endings in mammalian skin. Biol. Rev., 30, 159-195. 